УДК 622.233 .6

\author{
А.Д. Учитель ${ }^{1}$, Ю.А. Малиновский ${ }^{2}$, Д.П. Власенков ${ }^{2}$, И.Н. Кравчук ${ }^{2}$, \\ С.С.Терешина ${ }^{2}$
}

${ }^{1}$ Национальная металлургическая академия Украины

${ }^{2}$ Криворожский колледж Национального авиационного университета

\title{
ПРИЧИНЫ ВОЗНИКНОВЕНИЯ ДИНАМИЧЕСКОЙ НЕУСТОЙЧИВОСТИ БУРОВОГО СТАВА ПРИ РАБОТЕ СТАНКОВ СБШ-250
}

\begin{abstract}
Для проведения буровзрывных работ на ряде предприятий получили распространение буровые станки типа СБШ-250. Эксплуатация этих машин сопровождается интенсивными динамическими режимами. Такие ситуации возникают потому, что расчетные нагрузки, заложенные при проектировании, и схематизация бурового става, зачастую определялись без учета основных факторов и строились на необоснованных допущениях. Эти недостатки необходимо устранить на этапе создания новых станков и модернизации действующего бурового оборудования. Такую задачу позволит решить разработка и практическое применение единой методики инженерных расчетов по выбору конструктивных и технологических станков, обеспечивающих повышение надежности и долговечности инструмента, бурового става, а также механизмов вращения и подачи (буровых станков). В работе установлено, что вращающийся буровой став под действием крутящего момента и усилия подачи приобретает форму винтовой линии, и находится в динамически неустойчивом состоянии под действием переменных сил сопротивления со стороны забоя. В результате исследований выявлен источник динамически неустойчивого состояния става, которым является переменный характер фрикционного взаимодействия инструмента и породы. На эти эффекты накладываются начальные геометрические неправильности става и вызванные или центробежные силы инерции. При этом поперечные и продольные колебания обусловлены крутильными, и все они будут связаны. Во время работы става динамическая неустойчивость машины существенно возрастает, если угловая скорость вращения совпадает с какой либо собственной частотой изгибных колебаний става. Для устранения опасных зон неустойчивости необходимо действующие и проектируемые буровые станки укомплектовать автоматическими регуляторами, настроенными на оптимальное сочетание крутящего момента, продольной силы и скорости вращения става.
\end{abstract}

DOI: $10.34185 / 0543-5749.2020-2-74-93$

\section{Введение}

Для успешного конструирования и модернизации современных высокопроизводительных буровых станков шарошечного бурения и бурового инструмента к ним, необходимо разработать единую методику инженерных расчетов бурового става, бурового инструмента, вращателя и механизма подачи. В настоящее время существует большое количество методик расчета бурового става и всего станка в целом. Однако эти методики желательно систематизировать и изложить в них основные расчетные принци- пы при работе гибкого длинного стержня кольцевого сечения на совместное действие продольных, изгибающих и крутящих силовых факторов (рис. 1).

В такой методике необходимо представить источник возникновения опасных колебаний, оценить параметры бурового става, которые являются критичными для нормальной работы станка, определить зоны устойчивости основных колебательных процессов, а также наметить пути устранения или снижения уровня опасных самовозбуждающихся колебаний. 

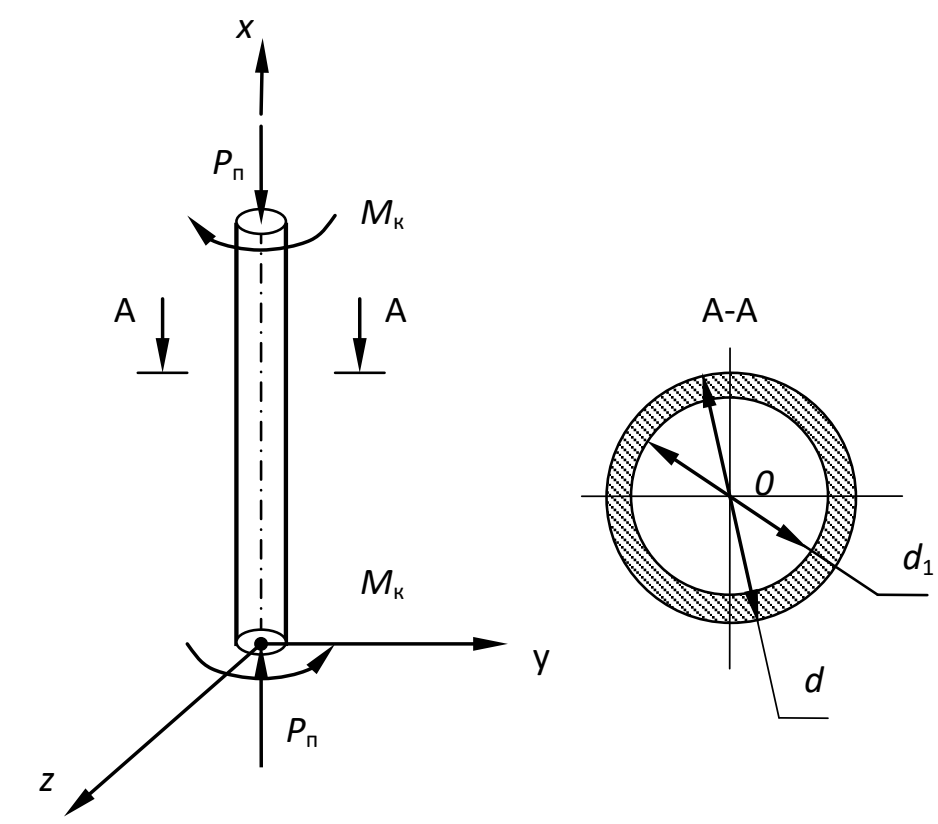

Pис. 1. Одновременное действие на бурильную колонну усилия подачи $P_{\text {п }}$ и крутящего момента $M_{\kappa}$ : А-А - поперечное сечение штанги

\section{Обзор литературы}

До настоящего времени не созданы достоверные глубоко обоснованные инженерные методики для расчетов станков шарошечного бурения с учетом динамических режимов и устойчивости в их работе.

Для обеспечения надежной работы буровых станков в разное время использовались различные гипотезы и предпосылки, которые до конца не дают возможности достоверно решить поставленные задачи. При этом ряд исследователей считают, что буровой став является длинным стержнем, подверженным продольной деформации, содержащим на концах две сосредоточенные массы и нагруженным импульсными нагрузками, и если частота повторения импульсов совпадает со временем внедрения двух соседних зубцов в забой, то так последовательно происходит раскачка продольных колебаний [1]. Другие исследователи ориентируются на явление продольного изгиба, формулу Эйлера и потерю ставом продольной устойчивости [2]. Третья группа исследователей считает, что буровой став изготавливается с начальными неправильностями (с эксцентриситетом). Вращение такого става приводит к развитию довольно значительных центробежных сил, что в ряде случаев является причиной дополнительных вибраций, снижения надежности и долговечности станка [3].

Из проанализированных источников следует, что нагрузки, заложенные при проектировании буровых станков, определяются со значительными погрешностями, которые необходимо устранить на этапе их модернизации. Кроме того, действующие и проектируемые буровые станки должны быть укомплектованы устройствами по регулированию оптимальных режимов работы по параметрам: усилие подачи, угловая скорость вращения става, крутящий момент.

\section{Изложение основного материала}

Рассмотрим равновесие бурового става, потерявшего устойчивость под действием крутя-

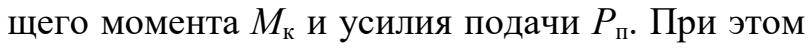
буровая колонна примет вид винтовой линии. Буровой став в недеформированном состоянии связан с неподвижной системой координат.

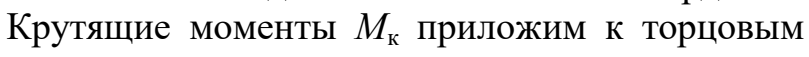
сечениям става, а продольные силы направим по вертикальной оси колонны $O x\left(P_{\text {п }}\right)$. В результате приложения к колонне значительных усилий 
произойдет искривление продольной оси става, а также произойдет разворот сечений става на определенные углы (рис. 1).

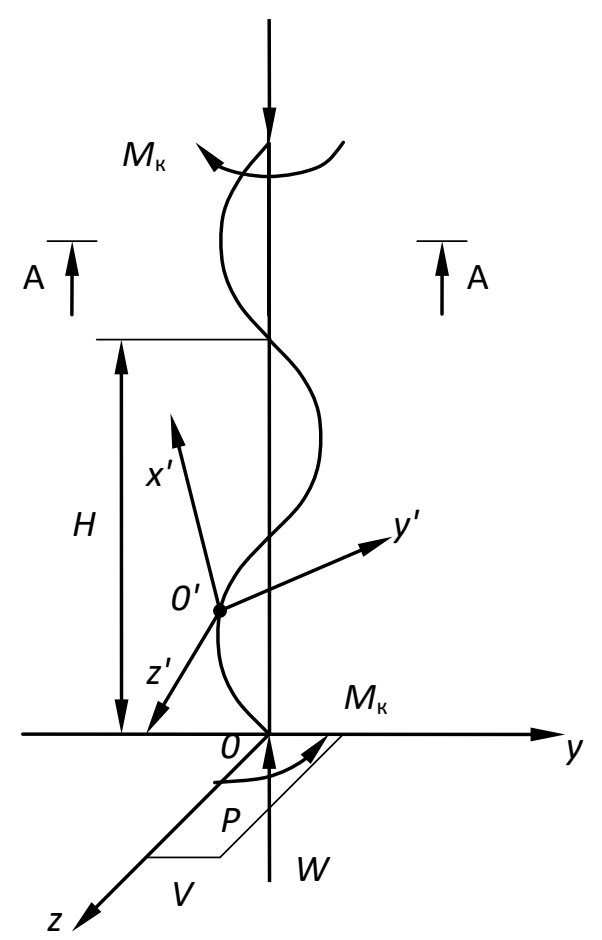

Рис. 2. Выпучивание бурильной колонны под

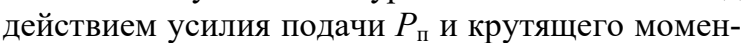
та $M_{\text {к }}$ a) $V, W$ - смещение точек оси бурового става при его деформации; б) $H$ - шаг винтовой линии при выпучивании бурового става; в) $0, x$, $y, z$ - основная система координат; г) $0^{\prime}, x^{\prime}, y^{\prime}, z^{\prime}-$ подвижная система координат, связанная с точками деформированной осью става

Введем в точке $\mathrm{O}^{\prime}$ некоторую систему координат $O^{\prime} x^{\prime} y^{\prime} z^{\prime}$, связанную с деформированной осью става. Проектируя силовые факторы на оси, связанные с поперечным сечением става, проходящем через начало координат, получим систему уравнений равновесия става (без учета динамических составляющих нагрузки) [4].

$$
\left\{\begin{array}{l}
E I \frac{d^{2} V}{d x^{2}}=-P_{n} V+M_{k} \frac{d W}{d x} \\
E I \frac{d^{2} W}{d x^{2}}=-P_{n} W-M_{k} \frac{d V}{d x}
\end{array},\right.
$$

где $E$ - модуль упругости материала штанг; $I-$ момент инерции поперечного сечения бурильной трубы (осевой); $M_{\text {к }}$ крутящий момент, приво-
Рассмотрим некоторую точку $O^{\prime}$ на пространственной кривой, которая представляет изогнутую ось става (рис. 2).

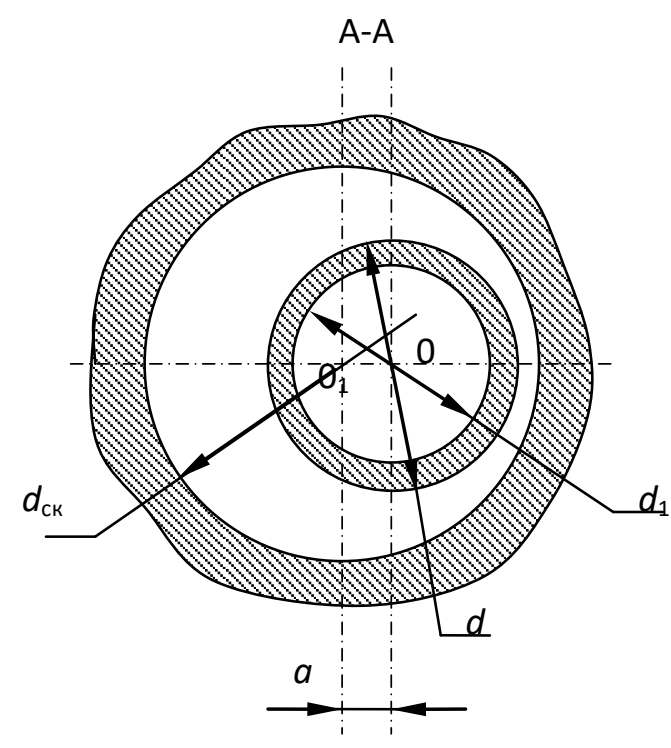

Рис. 3. Смещение оси штанги в скважине при выпучивании бурильной колонны: $d_{\text {ск }}-$ диаметр скважины; $a$ - смещение оси

дящий во вращение долото через буровой став.

$$
M_{k}=M_{k o}+\Delta M_{k},
$$

$M_{k o}$ - номинальное значение крутящего момента на вращателе; $\Delta M_{k}-$ приращение крутящего момента на буровом инструменте и вращателе, обусловленное непостоянным сопротивлением при трении бурового инструмента о разрушаемую породу и значительной крутильной податливостью бурового става; $P_{\text {п }}$ осевое усилие, сжимающее бурильную колонну, в общем случае переменная величина, связанная с воздействием горной породы (при соударениях и отскоках) на шарошечное долото.

$$
P_{n}=P_{n o}+\Delta P_{n},
$$


$P_{n o}-$ номинальное усилие подачи; $\Delta P_{n}-$ приращение усилия подачи за счет динамической составляющей при совместных крутильных, продольных и поперечных колебаниях става.

Систему уравнений равновесия бурового става будем рассматривать при граничных условиях:

1. Шарнирно опертый буровой став

$$
\begin{cases}\left.V\right|_{x=0}=0 ; & \left.V\right|_{x=l}=0 \\ \left.W\right|_{x=0}=0 ; & \left.W\right|_{x=l}=0 \ldots\end{cases}
$$

2. Став шарнирно оперт на вращателе, а в месте внедрения бурового става в забой имеет смещение $\left.V\right|_{x=l}=a$, при этом $\left.W\right|_{x=l}=0$ (так как буровой став после потери продольной устойчивости имеет форму винтовой линии, навитой на пробуренную скважину). При бурении скважины в месте внедрения инструмента в забой нет поперечных связей, удерживающих став (в состоянии шарнирного опирания).

$$
\left\{\begin{array}{l}
\left.V\right|_{x-0}=0 ;\left.\quad V\right|_{x-0}=a \\
\left.W\right|_{x-0}=0 ;\left.\quad W\right|_{x-0}=0 \ldots
\end{array}\right.
$$

После введения обозначений $\frac{M_{k}}{E I}=k$; $\frac{P_{n}}{E I}=S^{2}$; систему уравнений (1) преобразуем к виду (4). При этом решение систем вида (1), (4) выполним по методу, изложенному в [5].

$$
\left\{\begin{array}{l}
\frac{d^{2} V}{d x^{2}}+k \frac{d W}{d x}+S^{2} V=0 \\
\frac{d^{2} W}{d x^{2}}-k \frac{d V}{d x}+S^{2} W=0 .
\end{array}\right.
$$

Характеристическое уравнение системы (4) имеет вид:

$$
r^{2}+k r-\mathrm{s}^{2}=0,
$$

а его корни $r_{1}$ и $r_{2}$ равны

$$
r_{1,2}=-\frac{k}{2} \pm \sqrt{\frac{k^{2}}{4}+s^{2}} .
$$

Далее решение системы (4) представим в виде линейной комбинации тригонометрических функций для $V(x), W(x)$.

$$
\left\{\begin{array}{l}
V(x)=A \sin r_{1} x+B \cos r_{1} x+C \sin r_{2} x+D \cos r_{2} x \\
W(x)=-A \cos r_{1} x+B \sin r_{1} x-C \cos r_{2} x+D \sin r_{2} x .
\end{array}\right.
$$

Вычислим значения произвольных постоянных в выражении (7).

Рассмотрим сначала граничные условия 2 , если $x=0$, то $B+D=0 ; D=-B ; A+C=0$; $C=-A$.

Обратимся к граничным условиям при $x=1$ :

$$
\begin{aligned}
& \left\{\begin{array}{l}
A \sin r_{1} l+B \cos r_{1} l+C \sin r_{2} l+D \cos r_{2} l=a \\
-A \cos r_{1} l+B \sin r_{1} l-C \cos r_{2} l+D \sin r_{2} l .
\end{array}\right. \\
& A \sin r_{1} l+B \cos r_{1} l+C \sin r_{2} l+D \cos r_{2} l=a \\
& A\left(-\cos r_{1} l+\cos r_{1} l\right)+B\left(\sin r_{1} l-\sin r_{2} l=0\right. \text {. }
\end{aligned}
$$

Обозначая скобки через $\Delta_{1}, \Delta_{2}$ найдем коэффициенты для (7)

$$
\begin{gathered}
A \Delta_{1}+B \Delta_{2}=a ; \quad A+B \frac{\Delta_{2}}{\Delta_{1}}=\frac{a}{\Delta_{1}} ; \quad B\left(\frac{\Delta_{2}}{\Delta_{1}}+\frac{\Delta_{1}}{\Delta_{2}}\right)=\frac{a}{\Delta_{1}} ; \\
A \Delta_{2}+B \Delta_{1}=0 ; \quad-A+B \frac{\Delta_{1}}{\Delta_{2}}=0 ; \\
B\left(\frac{\Delta_{2}^{2}+\Delta_{1}^{2}}{\Delta_{1} \Delta_{2}}\right)=\frac{a}{\Delta_{1}} ; \quad B \frac{\Delta_{2}^{2}+\Delta_{1}^{2}}{\Delta_{2}}=a ; \quad B=\frac{a \Delta_{2}}{\left(\Delta_{1}^{2}+\Delta_{2}^{2}\right)} ; \\
A=B \frac{\Delta_{1}}{\Delta_{2}}=a \frac{\Delta_{1}}{\left(\Delta_{1}^{2}+\Delta_{1}^{2}\right)} .
\end{gathered}
$$

Запишем выражения для смещений произвольной точки бурового става в случае граничных условий 2 . Нас в большей степени интересуют смещения става в точке $x=l$, в зоне взаимодействия инструмента с забоем, тогда будем иметь:

$$
\begin{aligned}
& V(x)=a \frac{\Delta_{1}}{\Delta_{1}^{2}+\Delta_{2}^{2}} \sin r_{1} x+a \frac{\Delta_{2}}{\Delta_{1}^{2}+\Delta_{2}^{2}} \cos r_{1} x- \\
& -a \frac{\Delta_{1}}{\Delta_{1}^{2}+\Delta_{2}^{2}} \sin r_{1} x-a \frac{\Delta_{2}}{\Delta_{1}^{2}+\Delta_{2}^{2}} \cos r_{2} x, \\
& W(x)=-a \frac{\Delta_{1}}{\Delta_{1}^{2}+\Delta_{2}^{2}} \cos r_{1} x+a \frac{\Delta_{2}}{\Delta_{1}^{2}+\Delta_{2}^{2}} \sin r_{1} x+ \\
& +a \frac{\Delta_{1}}{\Delta_{1}^{2}+\Delta_{2}^{2}} \cos r_{2} x-a \frac{\Delta_{2}}{\Delta_{1}^{2}+\Delta_{2}^{2}} \sin r_{2} x . \\
& \text { Для } x=l V(l)=a ; W(l)=0 \\
& V(x)=a \frac{\Delta_{1}}{\Delta_{1}^{2}+\Delta_{2}^{2}}\left(\sin r_{1} x-\sin r_{2} x\right)+ \\
& +a \frac{\Delta_{2}}{\left(\Delta_{1}^{2}+\Delta_{2}^{2}\right)}\left(\cos r_{1} x-\cos r_{2} x\right),
\end{aligned}
$$




$$
\begin{aligned}
W(x)= & a \frac{\Delta_{1}}{\Delta_{1}^{2}+\Delta_{2}^{2}}\left(\cos r_{2} x-\cos r_{1} x\right)+ \\
& +a \frac{\Delta_{2}}{\left(\Delta_{1}^{2}+\Delta_{2}^{2}\right)}\left(\sin r_{1} x-\sin r_{2} x\right) .
\end{aligned}
$$

Рассмотрим граничные условия 1 при $x=l$ :

$$
\left\{\begin{array}{l}
A \sin r_{1} l+B \cos r_{2} l+C \sin _{2} l+D \cos _{2} l \\
-A \cos r_{1} l+B \sin r_{2} l+C \cos _{2} l+D \sin _{2} l,
\end{array}\right.
$$

поскольку $A+C=0(C=-a) ; B=D=0 ; D=-B$, то запишем:

$$
\begin{gathered}
A \Delta_{1}+B \Delta_{2}=0, \\
\mathrm{~A}\left(-\Delta_{2}\right)+\mathrm{B} \Delta_{1}=0 . \\
\left\{\begin{array}{l}
A+B \frac{\Delta_{2}}{\Delta_{1}}=0 \\
-A+B \frac{\Delta_{1}}{\Delta_{2}}=0 \quad B\left(\frac{\Delta_{1}}{\Delta_{2}}+\frac{\Delta_{2}^{2}+\Delta_{1}^{2}}{\Delta_{1} \Delta_{2}}\right)=0,
\end{array}\right.
\end{gathered}
$$

тогда $B=0 ; D=0$.

Таким образом, при нулевых граничных условиях 1 имеем:

$$
\left\{\begin{array}{l}
V(x)=A \sin r_{1} x-A \sin r_{2} x= \\
=A\left(\sin r_{1} x-\sin r_{2} x\right)=a\left(\sin r_{1} x-\sin r_{2} x\right) \\
W(a)=-A \cos r_{1} x+A \cos r_{2} x= \\
=-A\left(\cos r_{1} x-\cos r_{2} x\right)=-a\left(\cos r_{1} x-\cos r_{2} x\right) .
\end{array}\right.
$$

Принимаем, что постоянная $A=a$ (средний радиус бурового става, навитого на скважину) (рис. 3).

$$
\left\{\begin{array}{l}
V(x)=a\left(\sin r_{1} x-\sin r_{2} x\right) \\
W(x)=-a\left(\cos r_{1} x-\cos r_{2}\right) .
\end{array}\right.
$$

В приведенных выражениях $(7,8,9,10,11)$ $\Delta_{c}=\Delta_{1}^{2}+\Delta_{2}^{2}-$ определитель системы для вычислений неизвестных произвольных постоянных в решении системы уравнений (7).

Задача о потере продольной устойчивости става под действием продольного усилия подачи $P_{\mathrm{n}}$ и крутящего момента на вращателе $M_{\text {к }}$ представлена в статической постановке. Однако, как следует из опыта эксплуатации реальных буровых машин и проведенных лабораторных и промышленных экспериментов, работа таких машин обычно сопровождается значительными вибрациями бурового става и всего станка в целом [1].
Если решение уравнения (6) выразить через силовые факторы $M_{\mathrm{\kappa}}, P_{\mathrm{n}}$, то $\left(r_{1}-r_{2}\right)-$ разность корней уравнения будет равно $2 \pi / l$, где $l$ - является шагом винтовой линии, измеряемой по одной прямолинейной образующей. Тогда длина одной полуволны равна $l / 2=l_{\text {кр }}$, а подкоренное выражение преобразуем к виду (14).

Если $P_{\mathrm{n}} \equiv 0$, то $M_{\mathrm{\kappa}, \mathrm{kp}}=2 \pi \cdot E I / l$, т.е. став штанг может потерять устойчивость только от скручивающих пар.

Если $M_{\mathrm{K}} \equiv 0$, то это формула Эйлера при продольном изгибе стержня с двумя шарнирно закрепленными концами.

Критериальное уравнение

$$
\frac{M_{k}^{2}}{4(E I)^{2}}+\frac{P_{n}}{E I}=\frac{\pi^{2}}{l^{2}}
$$

можно разрешить относительно $l^{2}$, при этом если выполняется (14), то длина става будет равна критической длине $l=l_{\text {кр}}$, то став потеряет продольную устойчивость и приобретет форму винтовой линии. Преобразуем (14) к виду

$$
\frac{4 \pi^{2}(E I)^{2}}{M_{k}^{2}+4 P_{n}}=l^{2} \text {. }
$$

В этом случае $l=\lambda l_{\text {кр }}$, где $\lambda-$ коэффициент $0 \leq \lambda \leq n$, если $\lambda=0$, длина пробуренной скважины равна 0 , если $\lambda=1$, то став потеряет продольную устойчивость, изменит свою прямолинейную форму ( $\lambda_{\kappa}=1$ критическое значение коэффициента), если $\lambda=n$, то $l=l_{\max }$ - равна максимальной проектной длине скважины, и став потеряет устойчивость по всей длине скважины, причем число полуволн при изгибе и кручении оси става будет равно $n$, иначе став потеряет $n$ раз упругую устойчивость.

Выражения (14) или (15) являются критериальными уравнениями, однако по соотношению (14) невозможно сказать, какое из значений $M_{\text {к }}$ или $P_{\mathrm{n}}$ являются критическими. Состояние потери устойчивости бурового става наступает при определенных соотношениях $M_{\text {к }}$ и $P_{\mathrm{n}}$, поэтому нами для закрученного стержня постоянного сечения, нагруженного ещё и продольной нагрузкой вводится понятие «критической» длины става $l_{\text {кр }}$.

Отметим, что в системе уравнений (1), которая по форме записи является статической, введены приращения крутящего момента $\Delta M_{\text {к и }}$ продольной силы $\Delta P_{\mathrm{n}}$, характеризующие нестационарные процессы в буровом ставе при вы- 
полнении технологической операции бурения. Для определения этих приращений сформулируем предпосылки возникновения переменных нагрузок в ставе.

Одной из таких предпосылок, при постоянных силовых факторах в начале процесса, на наш взгляд, является нелинейное трение между обрабатываемой горной породой и буровым инструментом, и кроме того, податливый буровой став. Эти обстоятельства способствуют возникновению крутильных колебаний в бурильной колонне.

Нелинейное трение между горной породой и инструментом при переменной скорости скольжения инструмента приводит к ниспадающей характеристике трения, которая обеспечивает отрицательное демпфирование в упругой системе станка [6]. Переменная скорость скольжения инструмента обусловлена высокой податливостью бурового става и наличием в приводе двигателя ограниченной мощности. В те моменты времени, когда момент сил сопротивления бурению возрастает, а потенциальной энергии бурового става за счет его незначительной жесткости и недостаточной мощности двигателя не хватает для того, чтобы провернуть буровой инструмент с номинальной угловой частотой $\omega_{0}$, то фактическая угловая частота вращения инструмента резко уменьшается, в ряде случаев может иметь место полная остановка долота. В это время двигатель продолжает вращательное движение, при этом потенциальная энергия бурового става, как скручиваемой пружины, продолжает нарастать. Когда энергии бурового става бу- дет достаточно, произойдет раскрутка става и угловая скорость долота возрастает. Таким образом, возникают предпосылки для возникновения фрикционных, а в ряде случаев, и релаксационных крутильных колебаний в системе вращатель - буровой став - долото.

В дальнейшем, будем исходить из предположения, что сложные крутильные, продольные и изгибные колебания бурового става обусловлены нестационарным трением между буровым инструментом и забоем при изменении скорости проскальзывания между долотом и породой. В этом случае крутильные автоколебания приводят к продольным и в конечном случае к изгибным колебаниям бурового става. Поэтому дальнейший путь решения сводится к созданию расчетной схемы для описания этих колебаний и математической формулировке рассматриваемой задачи.

Для этого обратимся отдельно к крутильной колебательной системе станка, которую представим в виде двух масс, соединенных длинным валом (можно с распределенными параметрами). Для упрощения математической формулировки задачи ограничимся двухмассовой системой (рис. 4).

В качестве первой массы принимаем массу (момент инерции $I_{1}$ ) бурового долота с учетом части массы бурового става (до узла колебаний). В качестве второй массы принимаем массу (момент инерции $I_{2}$ ) ротора приводного электродвигателя с учетом части массы бурового става (от двигателя до узла крутильных колебаний).

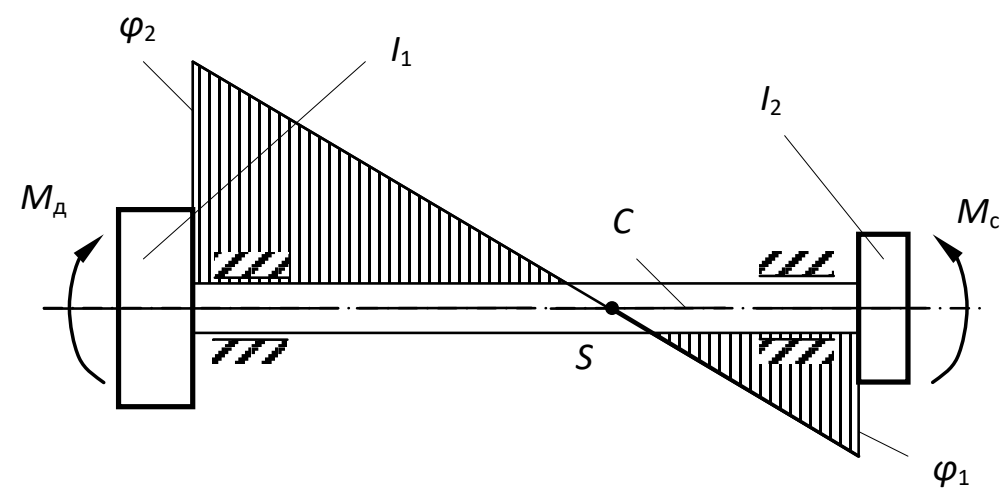

Рис. 4. Крутильная колебательная схема бурового станка: $I_{1}$ - момент инерции бурового инструмента; $I_{2}$ - момент инерции приводных механизмов; $C$ - крутильная жесткость става штанг; $S$ - узел колебаний; $\varphi_{1}, \varphi_{2}$ - максимальные углы закручивания мacc 
На каждую из масс действуют моменты сил инерции $-I_{2} \ddot{\varphi}_{2}-$ ротора двигателя и $-I_{1} \ddot{\varphi}_{1}-$ для бурового инструмента, момент сил упругости $C\left(\varphi_{2}-\varphi_{1}\right)$, момент приводного двигателя $M_{\text {дв }}$ и момент сил сопротивления со стороны забоя $M_{\mathrm{c}}$. Тогда система дифференциальных уравнений самовозбуждающихся крутильных колебаний для двухмассовой схемы принимает вид:

$$
\left\{\begin{array}{l}
I_{1} \ddot{\varphi}_{1}+C\left(\varphi_{1}-\varphi_{2}\right)=M_{c} \\
I_{2} \ddot{\varphi}_{2}+C\left(\varphi_{2}-\varphi_{1}\right)=M_{0}+M_{\partial}\left(\omega_{0}-\dot{\varphi}_{2}\right)
\end{array}\right.
$$

где $M_{c}$ - момент сил сопротивления при вращении бурового инструмента в массиве горной породы, по аналогии с работой [6] представим в форме (рис. 5)

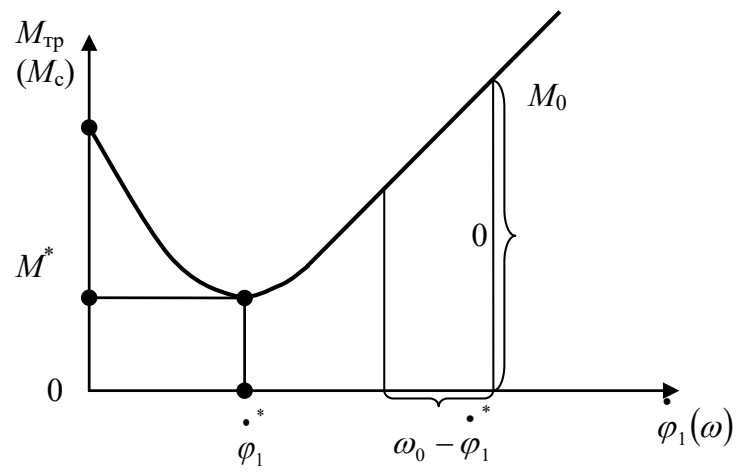

Рис. 5. Характеристика момента сил трения между буровым инструментом и породой, как функция скорости проскальзывания между забоем и инструментом

$M^{*}, \dot{\varphi}^{*}-$ критические значения момента сопротивления и угловой скорости вращения инструмента

$$
M_{c}=M_{\text {тр }}=M_{0}+\left(\frac{d M}{d \omega}\right)_{0}\left(\dot{\varphi}_{1}-\omega_{0}\right),
$$

где $\left(\frac{d M}{d \omega}\right)_{0}-$ значение производной при $\dot{\varphi}=\omega_{0}$ номинальной угловой скорости става; $C$ - крутильная жесткость бурового става (при бурении величина переменная); $C=\frac{G I_{p}}{l}=\frac{2 G I_{x}}{l} ; l-$ длина става (при бурении величина переменная); $G-$ модуль упругости (сдвига); $I_{p}=\frac{\pi}{32}\left(d^{4}-d_{1}^{4}\right)-$ полярный момент инерции сечения бурового става; $I_{X(Y)}=\frac{\pi\left(d^{4}-d_{1}^{4}\right)}{64}-$ осевой момент инерции сечения бурового става; $I_{1}-$ момент инерции массы бурового инструмента с учетом влияния части массы бурового става; $I_{2}-$ момент инерции массы ротора приводного двигателя с учетом влияния части массы бурового става; $M_{0}$ - номинальный вращающий момент электродвигателя (приведенной к оси бурового става); $M_{\text {д }}$ коэффициент демпфирования приводного электродвигателя; $\omega_{0}-$ номинальная угловая частота вращения ротора двигателя (става).
В рассматриваемом случае характеристика момента сил трения долота (бурового става) от скорости скольжения о забой имеет вид (рис. 5).

На участке характеристики (рис. 5) от 0 до $\dot{\varphi}_{1}^{*}$ имеет место отрицательное демпфирование (ниспадающий участок характеристики). На этой ветви характеристики в системе: вращатель - буровой став - инструмент имеют место незатухающие (авто)колебания. По мере бурения скважины жесткость става уменьшается и поэтому параметры колебательной системы и характеристики трения будут в некоторых пределах изменяться.

В целом, характеристика момента сил трения между забоем и инструментом носит нестационарный характер, имеет вид ниспадающей функции, и в определенных диапазонах изменения скоростей скольжения может способствовать возникновению автоколебаний в системе.

Для удобства анализа самовозбуждения колебаний будем полагать, что характеристика момента сил трения может быть представлена в виде [6]

$$
M_{c}=3 M^{*}\left(1-\frac{\Delta \dot{\varphi}_{1}}{\Delta \varphi_{1}^{*}}+\frac{\Delta \dot{\varphi}_{1}^{3}}{\Delta \dot{\varphi}_{1}^{* 3}}\right) .
$$

Выражение для момента сил трения (рис. 5) разложим по степеням $\Delta \dot{\varphi}_{1}$ : 


$$
\begin{aligned}
M_{c} & =M_{T P}=M_{0}+M_{1} \Delta \dot{\varphi}_{1}+ \\
& +M_{2} \Delta \dot{\varphi}_{1}^{2}-M_{3} \Delta \dot{\varphi}_{1}^{3}
\end{aligned},
$$

где $M_{0}, M_{1}, M_{2}, M_{3}$ - коэффициенты демпфирования:

$$
\begin{gathered}
M_{0}=3 M^{*}\left(1-\frac{\omega_{0}}{\Delta \dot{\varphi}_{1}^{*}}-\frac{\omega_{0}}{3 \Delta \dot{\varphi}_{1}^{* 3}}\right), \\
M_{1}=\frac{3 M^{*}}{\dot{\varphi}_{1}^{*}\left(1-\frac{\omega_{0}^{2}}{\Delta \dot{\varphi}_{1}^{2}}\right)}, \\
M_{2}=\frac{3 M^{*}}{\Delta \dot{\varphi}_{1}^{* 3} \omega_{0},} \\
M_{3}=\frac{M^{*}}{\Delta \dot{\varphi}_{1}^{* 3}} ; \Delta \dot{\varphi}_{1}=\dot{\varphi}_{1}-\omega_{0} .
\end{gathered}
$$

Решение системы (16) представлено в [7], где вместо функции $M_{C}(19)$ записана некоторая явная функция времени. Для того, чтобы решить непосредственно (16) с учетом (19) необходимо выполнить линеаризацию системы. Поэтому дальнейшее решение системы (16) будем строить исходя из упрощенного представления о том, что механизм вращатель - буровой став буровой инструмент обладает двигателем с жесткой характеристикой. Тогда вместо системы (16) будем иметь одно уравнение (20). После получения решения уравнения (20) результат можно подставить в систему (16) и получить уточненное решение (16) с учетом решения (20)

$$
\begin{gathered}
I_{1} \ddot{\varphi}_{1}+C\left(\varphi_{1}-\omega_{0} t\right)= \\
=M_{0}+M_{1} \Delta \dot{\varphi}_{1}+\Delta M_{2} \dot{\varphi}_{1}^{2}-\Delta M_{2} \dot{\varphi}_{1}^{3} .
\end{gathered}
$$

Введем следующие обозначения: $p^{2}=\frac{C}{I_{1}}-$ собственная частота крутильных колебаний системы буровой инструмент - бурильная колонна (приближенное значение); $\quad \bar{r}_{0}=\frac{M_{0}}{I_{1}}$; $\overline{r_{1}}=\frac{M_{1}}{I_{1}} ; \overline{r_{2}}=\frac{M_{2}}{I_{1}} ; \overline{r_{2}}=\frac{M_{2}}{I_{1}}-$ постоянные коэффициенты.

Тогда дифференциальное уравнение (20) преобразуем к виду:

$$
\begin{gathered}
\ddot{\varphi}_{1}-\bar{r}_{1} \Delta \dot{\varphi}_{1}-\bar{r}_{2} \Delta \dot{\varphi}_{1}^{2}+\bar{r}_{3} \Delta \dot{\varphi}_{1}^{3}+ \\
+p^{2} \varphi_{1}=\bar{r}_{0}+p^{2} \varphi_{0}^{t} .
\end{gathered}
$$

Рассмотрим дифференциальное уравнение
(21) в предположении, что скорость проскальзывания находится в области ниспадающего участка характеристики трения (рис. 5), при этом нелинейные слагаемые оказывают несущественное влияние и ими на первом этапе решения можно пренебречь, то есть пока остановимся на упрощенном уравнении:

$$
\ddot{\varphi}_{1}-\overline{r_{1}} \dot{\varphi}_{1}+p^{2} \varphi_{1}=\overline{r_{0}}+p^{2} \omega_{0} t .
$$

Составим характеристическое уравнение для дифференциального (22)

$$
\lambda^{2}-\overline{r_{1}} \gamma+p^{2}=0
$$

корни которого будут равны

$$
\lambda_{1,2}=\frac{\overline{r_{1}}}{2} \pm \sqrt{\frac{\overline{r_{1}^{2}}}{4}-p^{2}} .
$$

Полагаем, что $p^{2}>\frac{\overline{r_{1}^{2}}}{4}$, тогда корни

будут комплексно сопряженными и решение однородного уравнения для (22) получим в виде

$$
\widetilde{\varphi}_{1}(t)=e^{\overline{r_{1}} t / 2}\left(A_{1} \cos p_{1} t+B_{1} \sin p_{1} t\right),
$$

так как $\widetilde{\varphi}(0)=0 ; \dot{\widetilde{\varphi}}(0)=\omega_{0}$, то $A_{1}=0 ; B_{1} \neq 0$, тогда

$$
\begin{aligned}
& \widetilde{\varphi}_{1}(t)=e^{\overline{r_{1}} t} B_{1} \sin p_{1} t ; \dot{\tilde{\varphi}}_{1}(t)= \\
& =e^{\overline{r_{1} t}} B_{1} \sin p_{1} t+B_{1} p_{1} e^{\overline{r_{1}} t} \cos p_{1} t,
\end{aligned}
$$

где $p_{1}=\sqrt{p^{2}-\frac{\bar{r}_{1}^{2}}{4}} ; B_{1}=\frac{\omega_{0}}{p_{1}}$.

Частное решение (21) ищем в виде:

$$
\begin{aligned}
& \bar{\varphi}_{1}(t)=A_{2} t+B_{2} ; \dot{\bar{\varphi}}(0)=A_{2}=\omega_{0} ; \\
& \bar{\varphi}(0)=-\overline{r_{1}} \omega_{0}+p^{2} B_{2}=\overline{r_{0}} ; \\
& B_{2}=\frac{\overline{r_{0}}+\overline{r_{1}} \omega_{0}}{p^{2}} .
\end{aligned}
$$

Запишем общее решение (22) как сумму (26) и (27)

$$
\begin{gathered}
\varphi_{1}(t)=\widetilde{\varphi}_{1}(t)+\overline{\varphi_{1}}(t), \\
\varphi_{1}(t)=e^{\frac{\overline{r_{1}} t}{2} B_{1} \sin p_{1} t+\omega_{0} t+\frac{\overline{r_{0}}+\overline{r_{1}} \omega_{0}}{p^{2}}}
\end{gathered}
$$

или 


$$
\varphi_{1}(t)=e^{\overline{r_{1}} t} \frac{\omega_{0}}{p_{1}} \sin p_{1} t+\omega_{0} t+\frac{\overline{r_{0}}+\overline{r_{1}} \omega_{0}}{p^{2}}
$$

Таким образом, функция $\varphi_{1}(t)$ является приближенным решением системы уравнений (16) и (19). По виду решения следует, что в системе присутствуют гармонические нарастающие колебания с частотой собственных колебаний системы и отрицательным затуханием $\bar{r}_{1}$ (в линейной постановке).

Если автоколебания в системе происходит в нелинейной части характеристики трения, то для решения уравнения (21) необходимо дополнительно применить метод Ван-Дер Поля [6].

Согласно этому методу, автоколебания в системе являются квазигармоническими и происходят с переменной амплитудой

$$
B_{1}=B_{1}(t)=\frac{B_{0}}{\sqrt{\left(1-\frac{3 \overline{r_{2}} p_{1}^{2}}{4 \overline{r_{1}}} B_{0}^{2}\right) e^{-\overline{r_{1}} t}+\frac{3 \overline{r_{2}} p_{1}^{2}}{4 \overline{r_{1}}} B_{0}^{2}}} .
$$

В выражении (31) в качестве $B_{0}$ (начальной амплитуды) принимаем значение амплитуды из выражения (26)

$$
B_{0}=\omega_{0} / p_{1} .
$$

Если в формулу (29) подставить вместо $B_{1}$ выражение $B_{1}(t)$, то получим решение с учетом всех особенностей характеристики момента сил трения от скорости (рис. 5).

Поэтому в колебательной системе при работе в некоторой окрестности точки $\dot{\varphi}_{1}^{*}$ характеристики момента сил трения возникают автоколебания, однако они не будут носить нарастающий характер, так как при решении задачи учтены составляющие отрицательного и положительного демпфирования (коэффициенты $\overline{r_{1}}, \overline{r_{2}}$, вызывающие раскачку колебаний, а $\overline{r_{3}}-$ торможение. Полученное решение (30) с учетом выражения переменной амплитуды $B_{1}(t)$ подставим в правую часть первого уравнения системы (16) или в ее решение [7]. Таким образом, из вида решения (16), с учетом (19) и вида системы следует, что вследствие нестационарной характеристики момента $M_{\mathrm{C}}\left(M_{\text {тр }}\right)$ в механизме привод - буровой став - буровой инструмент возникают незатухающие крутильные колебания, обусловленные силами трения между буровым инструментом и породой. Эти колебания по частоте и форме напоминают свободные колебания в крутильной системе. Для описания этих процессов обратимся к системе (12) без правых частей. Рассмотрим при этом собственную частоту и законы колебания масс.

Предположим, что в момент рассмотрения система совершает незатухающие колебания без внешней нагрузки и сил сопротивления, а только при наличии начальной скорости $\dot{\varphi}_{0}$, таким образом система уравнений (12) будет однородной

$$
\left\{\begin{array}{l}
I_{1} \ddot{\varphi}_{1}+C\left(\varphi_{1}-\varphi_{2}\right)=0 \\
I_{2} \ddot{\varphi}_{2}+C\left(\varphi_{2}-\varphi_{1}\right)=0
\end{array}\right.
$$

Закон колебаний бурового инструмента при закручивании става можно представить в виде $\varphi_{1}(\mathrm{t})$, а закон колебаний ротора двигателя в виде $\varphi_{2}(\mathrm{t})$ :

$$
\begin{aligned}
& \varphi_{1}(t)=\frac{\dot{\varphi}_{0}\left(I_{1}-I_{2}\right)}{I_{1}+I_{2}} t-\frac{2 \dot{\varphi}_{0} I_{2}}{p_{1}\left(I_{1}+I_{2}\right)} \sin p_{1} t \\
& \varphi_{2}(t)=\frac{\dot{\varphi}_{0}\left(I_{1}-I_{2}\right)}{I_{1}+I_{2}} t+\frac{2 \dot{\varphi}_{0} I_{2}}{p_{1}\left(I_{1}+I_{2}\right)} \sin p_{1} t
\end{aligned}
$$

где $p_{1}=\sqrt{\frac{C\left(I_{1}+I_{2}\right)}{I_{1} I_{2}}}-$ собственная частота колебаний (крутильных) бурового става с учетом массы ротора приводного двигателя и массы бурового инструмента; $C$ - крутильная жесткость бурового става (в зависимости от его длины $l$ ) $C=G I_{p} / l ; \quad I_{\mathrm{P}}-$ полярный момент сопротивления сечения штанги.

Решение системы записано в предположении, что первая и вторая массы става $\left(I_{1}, I_{2}\right)$ получили начальную скорость $\dot{\varphi}_{0}$ (с разными знаками).

Введем очевидное равенство $\dot{\varphi}_{0}=\omega_{0}$, и дифференцируя выражения (34), (35) по времени, получим законы изменения угловых скоростей для первой и второй масс

$$
\begin{aligned}
& \omega_{1}(t)=\omega_{0} \frac{I_{1}-I_{2}}{I_{1}+I_{2}}-\frac{2 \omega_{0} I_{2}}{I_{1}+I_{2}} \cos p_{1} t \\
& \omega_{2}(t)=\omega_{0} \frac{I_{1}-I_{2}}{I_{1}+I_{2}}+\frac{2 \omega_{0} I_{2}}{I_{1}+I_{2}} \cos p_{1} t .
\end{aligned}
$$

Эти крутильные автоколебания в соответствии с системой уравнений (1) оказываются связанными с продольными колебаниями. При этом буровой став в состоянии близком к потере 
продольной устойчивости практически является винтовой пружиной, навитой на скважину. Характерной особенностью этой пружины является значительный шаг навивки «спирали» на выработку. Поэтому результаты многих исследований по статике и динамике пружин дают весьма приближенные результаты [8] для пружин с большим шагом витка.

Для определения приращения продольной силы $P_{n}=P_{0}=\Delta P_{n}$ поступим следующим образом. Вычисленные значения $v ; \frac{d v}{d x} ; \frac{d^{2} v}{d x^{2}} ; \frac{d w}{d x} ;$ формулы (10) и (11) подставим в первое уравнение системы (1), учитывая при этом, что $M_{k}=M_{k 0}+\Delta M_{k}$ и выразим $\Delta P_{n}$ через параметры системы и $\Delta M_{k}$ и граничные условия (2) или (3) при $x=l$

$$
\begin{aligned}
& \Delta P_{n}=-\frac{E I}{\Delta_{1}^{2}+\Delta_{2}^{2}}\left[\Delta_{1} r_{2}^{2} \sin r_{2} l-\Delta_{2} r_{1}^{2} \cos r_{1} l-\right. \\
& \left.-\Delta_{2} r_{2}^{2} \cos r_{2} l\right]+\frac{\Delta M_{k}}{\Delta_{1}^{2}+\Delta_{2}^{2}}\left[\Delta_{2} r_{1} \cos r_{1} l-\right. \\
& \left.-\Delta_{2} r_{2} \cos r_{1} l-\Delta_{1} r_{1} \sin r_{2} l+\Delta_{1} r_{1} \sin r_{1} l\right] .
\end{aligned}
$$

Переменная величина $\Delta M_{k}$ может быть выражена на основании формулы (19) в виде

$$
\Delta M_{k}=M_{1} \Delta \dot{\varphi}_{1}+M_{2} \Delta \dot{\varphi}_{1}^{2}-M_{3} \Delta \dot{\varphi}_{1}^{3},
$$

где $\Delta \dot{\varphi}_{1}(t)=e^{\overline{r_{1}}} B_{1}\left(\frac{\overline{\eta_{1}}}{2} \sin p_{1} t+p_{1} \cos p_{1} t\right) ; B_{1}-$ амплитуда автоколебаний, принимаем либо $B_{1}=\omega_{0} / p_{1}$, либо в виде (31) $B_{1}=B_{1}(t)$.

Таким образом, продольная сила (усилие подачи) содержит переменную составляющую, обусловленную переменным трением между забоем и буровым инструментом, изменяющуюся с частотой крутильной колебательной системы.

Следовательно, источником сложных продольно-изгибно-крутильных автоколебаний являются фрикционные эффекты на контакте буровой инструмент - забой.

При определении неустойчивых динамических состояний бурового става важным является вопрос вычисления динамических значений усилия подачи $P_{\text {пд }}$ и крутящего момента $M_{k}$, а также их динамических приращений $\Delta P_{n}, \Delta M_{k}$. Прием, использованный в (38) для определения динамического значения усилия подачи $P_{\text {пд},}$ требует громоздких вычислений и не учитывает величины свободного хода $(h)$ при продольных вибрациях става. Как показывает практика, продольные перемещения и продольные усилия при колебаниях могут изменяться в широких пределах. Вместе с тем, крутильные колебания в установившемся режиме «самовозбуждения» изменяются в пределах изменения коэффициента трения между породой и буровым инструментом. Поэтому в реальных машинах амплитуды крутильных «автоколебаний» ограничены и не носят нарастающего характера, а амплитуды продольных и поперечных колебаний являются в ряде случаев нарастающими, так как они связаны с неустойчивыми параметрическими колебаниями става.

Далее вычислим динамические значения усилий и моментов баланса энергий и работ при выполнении машиной рабочего цикла. Обратимся отдельно к случаю продольного удара долота со ставом о забой. При этом предположим, что работа по перемещению бурового става, как жесткого тела, при выборке свободного хода механизмом подачи с учетом его упругой деформации полностью идет на накопление ставом потенциальной энергии деформации.

Запишем уравнение баланса работ

$$
P_{n}\left(h+\delta_{\text {д }}\right)=\frac{E F \delta_{\text {д }}^{2}}{l},
$$

где $P_{\text {п }}$ - статическое усилие, развиваемое механизмом подачи; $h$ - перемещение бурового става, как сплошного тела (свободный ход, например, канатно-полиспастной системы); $\delta_{\text {д }}-$ динамическое укорочение (удлинение) под действием на став динамического усилия $P_{\text {д }} ; l-$ текущая длина с учетом удлинения при подаче става и его наращивании (в предположении, что став потерял упругую устойчивость); $E F$ - продольная жесткость бурового става; $E$ - модуль упругости первого рода для материала штанг; $F$ - площадь поперечного сечения буровой штанги.

Если ввести обозначение:

$$
\delta_{\text {ст }}=\frac{P_{n} l}{E F},
$$

где $\delta_{\text {ст }}$ - статическое укорочение става под действием номинального усилия подачи $P_{\text {п }}$ (имеет место до потери стержнем упругой устойчивости), тогда выражение (40) преобразуем к виду 


$$
\delta_{\text {д }}^{2}-2 \delta_{\text {д }} \delta_{\text {ст }}-2 \delta_{\text {ст }} h=0
$$

и динамическое укорочение запишем

$$
\delta_{\text {д }}=\delta_{\text {ст }}+\sqrt{\delta_{\text {ст }}^{2}+2 h \delta_{\text {ст }}} .
$$

Введем обозначение

$$
k_{\text {д }}=\delta_{\text {д }} / \delta_{\text {ст }} .
$$

Тогда с учетом теории соударения твердых тел [9] будем иметь

$$
k_{\text {д }}=1+\sqrt{1+\frac{2 h}{\delta_{\text {ст }}}} .
$$

Выражение (45) позволяет вычислять динамические усилия в буровом ставе до потери им упругой устойчивости

$$
P_{\mathrm{n}}=k_{\mathrm{n}} P_{\text {д }} .
$$

Рассматривая продольные колебания, из структуры выражения (45) следует, что коэффициент динамичности и амплитуды колебаний достигают значительных величин в силу того, что буровой став как жесткий объект имеет значительный свободный ход, который согласно [1] достигает значений $h \geq 50$ мм, и, если при этом статическое укорочение става составляет 5 6 мм (иногда до 8 мм), то коэффициент динамичности в системе превысит значение $k_{\mathrm{n}} \geq 5,5$.

Если же потеря устойчивости бурового става произошла, то перемещение става определим из условия, что ось бурового става представляет винтовую линию. Тогда по аналогии с винтовыми пружинами с большим шагом навивки [10] суммарное продольное укорочение (статическое) определим в соответствии с зависимостью

$$
\begin{aligned}
& \delta_{y c}=\delta_{1}+\delta_{2}\left[P_{n \kappa p} a^{2}\left(\frac{\sin ^{2} \alpha}{E I}+\frac{\cos ^{2} \alpha}{G l_{p}}\right)\right]+ \\
& +M_{k(k p)} a\left(\frac{1}{G l_{p}}+\frac{1}{E I}\right) \sin \alpha \cdot \cos \alpha \times \\
& \times \sqrt{\pi^{2} a^{2}+\frac{4 \pi^{2} E^{2} I^{2}}{M_{k(k p)^{2}}+4 P_{n \kappa p} E I}} \lambda,
\end{aligned}
$$

где $M_{\mathrm{k}(\mathrm{kp})}-$ величина критического крутящего момента; $P_{\mathrm{n}(\mathrm{kp})}-$ критическая продольная нагрузка бурового става; $a=\frac{d_{c k}-d_{1}}{2}-$ радиус оси цилиндрической винтовой линии, «навитой» на пробуренную скважину; $\alpha=H / 2 \pi \alpha-$ угол подъема витка винтовой линии; $H=2 l_{\mathrm{kp}}-$ шаг винтовой линии $\left(l_{\text {кр }}\right.$ - длина одной полуволны винтовой линии); $I_{p}=\frac{\pi d 4}{32}\left[1-\left(\frac{d_{1}}{d}\right)^{4}\right]-$ полярный момент инерции сечения штанги; $I=I_{x}=\frac{\pi d^{4}}{64}\left[1-\left(\frac{d_{1}}{d}\right)^{4}\right]-$ осевой момент инерции сечения штанги; $G$ - модуль сдвига материала штанги; $\lambda$ - число полуволн винтовой линии при потере ставом продольной устойчивости; $d, d_{1}-$ соответственно наружный и внутренний диаметры штанги.

Если став потерял прямолинейную форму и его изогнутая ось представляет собой пространственную кривую, то для определения коэффициента динамичности и динамического продольного усилия в ставе используем те же фор-

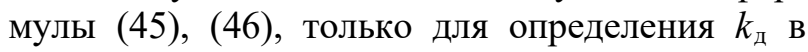
формуле (45) вместо $\delta_{\text {ст }}$ подставим величину $\delta_{\text {ус }}$ (47).

В этом случае коэффициент динамичности при продольных колебаниях става будет зависеть от параметров деформации оси става $(a, \alpha)$, а также обоих силовых факторов $P_{\text {п }}, M_{k}$. Отдельно рассмотрим динамический крутящий момент и коэффициент динамичности при закручивании става, так как природа динамического крутящего момента в случае рассмотрения крутильных колебаний будет иной.

Рассмотрим уравнения баланса кинетической и потенциальной энергий при неравномерном вращении става, на которое наложены крутильные колебания в виде

$$
I_{M} \cdot \frac{\left(\omega_{1}^{2}-\omega_{2}^{2}\right)}{2}=\frac{G I_{p} \varphi_{1 д}^{2}}{2 l}
$$

где $I_{M}=M \frac{d^{2}}{8}-$ момент инерции массы бурового инструмента с учетом части массы бурового става (до узла колебаний); $M$ - масса инструмента; $\omega_{1}, \omega_{2}-$ наибольшее и наименьшее значение угловой скорости бурового инструмента в режиме фрикционных автоколебаний; $\varphi_{1 д}=k_{\text {дк }} \varphi_{1 c \text { т }}-$ динамический угол закручивания конца бурового става у забоя (для массы $m_{1}$ ); $k_{\text {дк }}-$ коэффициент динамичности при кручении става; $\varphi_{1 \text { ст }}-$ статический угол закручивания конца става у забоя; $l$ - текущая длина бурового става. 
Рассмотрим предварительно левую часть уравнения энергетического баланса. При циклическом изменении угловой скорости бурового инструмента

$$
\left\{\begin{array}{l}
\omega_{1}=\omega_{0}+\Delta \omega \\
\omega_{2}=\omega_{0}-\Delta \omega
\end{array}\right.
$$

где $\omega_{0}$ - номинальная скорость вращения бурового става (установившееся её значение); $\Delta \omega-$ приращение угловой скорости за один цикл её изменения.

Выполнив несложные преобразования с учетом выражения (39), получим:

$$
I_{1} \frac{\omega_{1}^{2}-\omega_{2}^{2}}{2}=I_{1_{M}} \omega_{0} 2 \Delta \omega .
$$

При рассмотрении правой части (48) остановимся на величинах $\varphi_{\text {д }}, \varphi_{\text {ст, }} k_{\text {дk. }}$ Считая, что став потерял упругую устойчивость, его форма будет соответствовать винтовой линии, которая получила остаточные деформации под действием силовых факторов $P_{\text {п }}, M_{k}$.

По аналогии с [10] суммарный угол разворота концов става можно представить следующим образом

$$
\begin{aligned}
\varphi_{1 C M} & =\left[P_{\Pi} a\left(\frac{1}{G l_{p}}-\frac{1}{E l}\right) \sin \alpha \cdot \cos \alpha+\right. \\
& \left.+M_{k}\left(\frac{\cos ^{2} \alpha}{E I} \frac{\sin ^{2} \alpha}{G l_{p}}\right)\right] \times \\
& \times \sqrt{\pi^{2} a^{2}+\frac{4 \pi^{2} E I^{2}}{M_{k}^{2} 4 P_{\Pi} E I} \cdot \lambda .}
\end{aligned}
$$

Тогда выражение (48) приобретает вид:

$$
I_{1 M} \omega_{0} 2 \Delta \omega=\frac{G I_{p} \varphi_{1 d}^{2}}{2 l},
$$

или

$$
\begin{aligned}
& I_{1 M} \omega_{0} 2 \Delta \omega=k_{\text {дк }}^{2} \frac{G I_{p} \varphi_{1 \mathrm{c \tau}}^{2}}{2 l} \\
& k_{\text {мд }}=\frac{I_{1 M} \omega_{0} 2 \Delta \omega 2 l}{G I_{p} \varphi_{1 \mathrm{cт}}^{2}}
\end{aligned}
$$

Для определения коэффициента динамичности при закручивании става и потере им упругой устойчивости в формулу (54) подставляем вместо $\varphi_{1 \text { ст }}$ выражение (51). Если став не поте- рял упругой устойчивости, то $\varphi_{1_{\mathrm{c \tau}}}=\frac{M_{k} l}{G I p}$ определяем по упрощенной формуле [11]. Остановимся на выражении $\Delta \omega$ - это амплитуда колебаний угловой скорости бурового инструмента из выражения (55). Фактически угловая скорость $\omega$ колеблется около своего номинального (среднего) значения по закону

$$
\dot{\varphi}_{1}(t)=e^{\frac{\bar{\eta}_{1}}{2} \cdot t} B_{1}\left(\frac{\bar{y}_{1}}{2} \sin p_{1} t+p_{1} \cos p_{1} t\right)+\omega_{0},
$$

где $\Delta \omega=\frac{\omega_{0}}{p_{1}} \sqrt{\frac{\bar{\eta}}{4}+p_{1}^{2}}-$ амплитуда колебаний угловой скорости става.

Внося значение для $\Delta \omega$ в выражение (54), получим выражение для коэффициента динамичности при крутильных колебаниях става

$$
\begin{aligned}
k_{\mathrm{Mд}} & =\sqrt{\frac{2 I_{1} \omega_{0}^{2} l \sqrt{\bar{r}_{1}^{2} / 4+p_{1}^{2}}}{p_{1} G I_{p} \varphi_{1 \mathrm{cT}}^{2}}}= \\
& =\frac{2 I_{1 \mathrm{M}} \omega_{0}^{2} l \sqrt{\bar{r}_{1}^{2} / 4+p_{1}^{2}}}{p_{1} G I_{p} \varphi_{1 \mathrm{cT}}^{2}} .
\end{aligned}
$$

Если допустить, что динамичность системы при крутильных колебаниях обусловлена только крутящим моментом, то вместо формулы (56) используем формулу:

$$
k_{\text {мd }}=\frac{2 \omega_{0}}{M_{k}} \sqrt{\frac{I_{1 M} G I_{p}}{p_{1} l} \sqrt{\bar{r}_{1}^{2} / 4+p_{1}^{2}}} .
$$

В приведенных выражениях (56), (57): $\omega_{0}-$ номинальная (установившаяся) скорость вращения бурового става; $G$ - модуль упругости II рода для материала става; $I_{p}$ - полярный момент инерции поперечного сечения штанги; $M_{k}-$ номинальное значение крутящего момента на вращателе; $\overline{r_{1}}-$ коэффициент характеристики трения бурового инструмента о забой, который соответствует отрицательному демпфированию для нисходящей ветви характеристики $\left(\overline{r_{1}}={ }^{M_{1}} / I_{1}\right) ; I_{1 \mathrm{M}}-$ момент инерции массы инструмента с учетом части массы бурового става; $p_{1}-$ собственная частота двухмассовой крутильной колебательной системы вращатель (с приводом) - буровой став - инструмент; $l$ - текущая длина става (с учетом демпфирования при трении инструмента о забой и тяговой характеристики двигателя). 
После определения динамических составляющих крутящего момента $\Delta M_{k}$ и продольной силы $\Delta P_{n}$, а также после оценки динамических составляющих по сравнению со статическими, с помощью коэффициентов динамичности , обратимся к вопросу о продольных колебаниях, возникающих в ставе при выполнении буровых работ. Величина $\Delta P_{n}$ является нелинейной квазигармонической функцией времени вида (38). Тогда усилие подачи с учетом её гармонической составляющей представим в виде:

$$
P_{n}=P_{n 0}+\Delta P_{n},
$$

или представим в стандартной форме (в форме Матье) при описании продольно-поперечных колебаний для состояния, близкого к потере ставом продольной устойчивости

$$
P_{n}=P_{0}+P_{t} \cos \mathrm{p}_{1} t .
$$

Как следует из работ [3], буровой став СБШ250 , набираемый из четырех штанг длиной 8 м каждая, имеет начальный эксцентриситет или начальное искривление. Если поперечный изгиб става обозначить через $v(x, t)$, то начальное искривление обозначим $v_{0}(x)$. Для составления дифференциального уравнения динамической продольной устойчивости сжатого стержня (кроме того, подверженного закручиванию), исходим из уравнения статического изгиба перед потерей им продольной устойчивости [9], дважды его дифференцируем и добавляем силы инерции $-m \frac{\partial^{2} v}{\partial t^{2}}$ и, кроме того вместо продольной си-

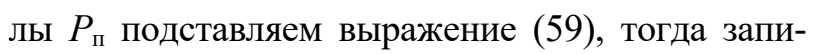
шем уравнение для динамической продольной устойчивости (однородная форма записи) и добавим в правую часть выражение для дополнительного изгибающего момента, вызванного начальной неправильностью става штанг

$$
\begin{aligned}
& E I \frac{\partial^{4} v}{\partial x^{4}}+\left(P_{0}+P_{t} \cos p_{1}\right) \frac{\partial^{2} v}{\partial x^{2}}+ \\
& +m \frac{\partial^{2} v}{\partial x^{2}}=-\left(P_{0}+P_{t} \cos p_{1} t\right) \frac{\partial^{2} v_{0}}{\partial x^{2}} .
\end{aligned}
$$

При составлении уравнения колебаний не учитываем пространственную форму изогнутой оси става, так как эта форма проявляется на значительной длине. В этом случае учет пространственной формы существенно усложнит задачу. Учитывая шарнирное опирание става как длинного стержня во вращателе и забое, представим форму изогнутой оси и форму начальной неправильности в виде синусоид

$$
\begin{aligned}
& v(x, t)=v(t) \sin \frac{\pi}{l} x, \\
& v_{0}(x)=v_{0} \sin \frac{\pi}{l} x .
\end{aligned}
$$

После подстановки выражений (61), (62) в (60) и сокращения подобных по аналогии с [9], приходим к уравнению

$$
\begin{aligned}
& v^{\prime \prime}+\Omega^{2}\left(1-2 \mu \cos p_{1} t\right) v= \\
& =\frac{\left(\omega^{2} v_{0}\right)}{P_{*} \pi} \cdot\left(P_{0}+P_{t} \cos p_{1} t\right) .
\end{aligned}
$$

В ряде расчетных случаев необходимо учитывать эксцентриситет приложения нагрузки к инструменту става, в нашем случае $e \equiv a$, тогда дополнительный изгибающий момент запишем в виде

$$
M=P_{n} \cdot a .
$$

Разложим изгибающий момент в ряд по собственным формам изгиба става

$$
M=\frac{4 P_{n} a}{\pi} \sum_{k=1,3,5 \ldots}^{\infty} \frac{1}{k} \sin \frac{k \pi x}{l} .
$$

Учитывая малость величины $e \equiv a$ по отношению к длине одной штанги, ограничимся $k=1$, запишем тогда выражение для эквивалентной кривизны (начальной неправильности) става

$$
v_{0}=4 a / \pi \text {. }
$$

Тогда для случая приложения продольной нагрузки к долоту с эксцентриситетом запишем (63) в виде

$$
\begin{aligned}
& v^{\prime \prime}+\Omega^{2}\left(1-2 \mu \cos p_{1} t\right) v= \\
& =\frac{\omega^{2} 4 a}{P_{*} \cdot \pi}\left(P_{0}+P_{t} \cos p_{1} t\right) .
\end{aligned}
$$

Обращаясь к выражению (66), получим значение коэффициента в правой части уравнения с учетом $v_{0}=a$ :

$$
\gamma=\frac{\omega^{2} 4 a}{P_{*} \pi}>\frac{\omega^{2} a}{P_{*}} .
$$

Следовательно, случай приложения нагрузки к буровому инструменту с эксцентриситетом является более опасным, чем начальная неправильность в пределах $0 \leq v_{0} \leq a$. 
Если добавить в одно из уравнений параметрических колебаний слагаемые, учитывающие затухание, нелинейную упругость, нелинейное затухание и нелинейную инерционность, то вместо уравнений (63), (67) будем иметь:

$$
\begin{aligned}
v^{\prime \prime}+2 \varepsilon v^{\prime} & =\Omega^{2}\left(1-2 \mu \cos p_{1} t\right)+\psi\left(v, v^{\prime}, v^{\prime \prime}\right)= \\
& =\frac{\omega^{2} v_{0}}{P_{*} \pi}\left(P_{0}+P_{t} \cos p_{1} t\right) .
\end{aligned}
$$

В приведенных выражениях (63), (67), (69): $\varepsilon-$ коэффициент линейного затухания системы, пропорциональный скорости продольных деформаций става; $\Omega_{k}=\omega_{k} \sqrt{1-\frac{P_{0}}{P_{*_{k}}}}-$ частота собственных колебаний стержня (става), загруженного постоянной составляющей продольной силы $(k=1,2,3,4 \ldots) ; P_{*}-$ критическая сила (по Эйлеру); $P_{*}=\frac{\pi^{2} E I}{l^{2}} ; m-$ масса става, отнесенная к единице длины; $\mu$ - коэффициент возбуждения нагрузки $\mu=\frac{P_{t}}{2 P_{*}-P_{0}} ; P_{t}-$ амплитуда колебаний продольной нагрузки; $\psi\left(v, v^{\prime}, v^{\prime \prime}\right)-$ некоторая нелинейная функция продольного перемещения, скорости и ускорения става.

Заметим, что в ряде случаев нагружения бурового става вид функции продольной нагрузки может быть другой

$$
P_{n}=P_{0}+P_{t} \Phi(t)
$$

где $P_{t} \Phi(t)$ - некоторая периодическая функция времени (например, пульсирующая или импульсивная функция), где $T=\left(2 \pi / P_{1}\right)-$ период функции; $p_{1}$ - частота колебаний в крутильной двухмассовой системе станка.

Если в уравнение (69) ввести значение $P_{n}$ из (70), то получим обобщенный случай уравнения Матье - уравнение Матье-Хилла [9].

$$
\begin{aligned}
v^{\prime \prime}+2 \varepsilon v^{\prime} & +\Omega^{2}(1-2 \mu \Phi(t))+\psi\left(v, v^{\prime} v^{\prime \prime}\right)= \\
& =\frac{\omega^{2} v_{0}}{P_{*} \pi}\left(P_{0}+P_{t} \Phi(t)\right) .
\end{aligned}
$$

Укажем частоты, при которых могут иметь место области неустойчивости при решении уравнений, описывающих параметрические колебания (67), (69), (71)

$$
\Theta_{*}=\frac{2 \Omega}{k}, \quad k=1,2,3, \ldots
$$

То есть зоны вблизи частот собственных колебаний стержня $\Omega$, отличающиеся от них в целое число раз, представляют собой зоны неустойчивых колебаний.

Так как частота колебаний продольной силы связана с частотой крутильных колебаний $p_{\mathrm{k}}$, то формула для определения частот из областей неустойчивости в нашем случае запишется так:

$$
p_{k}=\frac{2 \Omega}{k}, \quad k=1,2,3, \ldots
$$

То есть, параметрический резонанс в системе буровой инструмент - став - вращатель будет иметь место, если частота собственных изгибных колебаний става, загруженного постоянной продольной силой $\left(P_{0}\right)$ составляет $2 / \kappa$ часть от собственной частоты $\Omega$.

Для решения задачи (67) ограничимся гармоническим приближенным решением:

$$
v(t)=b_{0}+a_{2} \sin p_{1} t+b_{2} \cos p_{1} t .
$$

Если нелинейность системы (67) или (71) не очень велика, то можно воспользоваться решением линейной задачи [9]:

$$
\left\{\begin{array}{l}
b_{0}=\mu b_{2}=\frac{v_{0} P_{0}}{P_{*}-P_{0}} \\
\left(1-n^{2}\right) a_{2}-\frac{n \Delta}{\pi} b_{2}=2 v_{0} \mu \\
\left(1-n^{2}\right) b_{2}-2 \mu b_{0}+\frac{n \Delta}{\pi} a_{2}=0 .
\end{array}\right.
$$

где $\mu$ - коэффициент возбуждения нагрузки.

Введем обозначения коэффициентов влияния $n=\frac{p_{1}}{\Omega} ; \quad \Delta=\frac{2 \pi \varepsilon}{\Omega}$.

Из системы (75) находим коэффициенты гармонического разложения искомой функции.

Из решения системы следует:

$$
\begin{gathered}
b_{2}=\frac{v_{0} P_{0}}{P_{*}-P_{0}}+ \\
+\frac{1-n^{2}}{\left(1-n^{2}\right)\left(1-n^{2}-2 \mu^{2}\right)+\left(\frac{n \Delta}{\pi}\right)} \cdot \frac{2 \mu^{2} v_{0}}{1-\frac{P_{0}}{P_{*}}}, \\
b_{2}=\frac{1-n^{2}}{\left(1-n^{2}\right)\left(1-n^{2}-2 \mu^{2}\right)+\left(\frac{n \Delta}{\pi}\right)} \times \\
\times \frac{2 \mu^{2} v_{0}}{1-\frac{P_{0}}{P_{*}}},
\end{gathered}
$$




$$
\begin{gathered}
a_{2}=\frac{\frac{n \Delta}{\pi}}{\left(1-n^{2}\right)\left(1-n^{2}-2 \mu^{2}\right)+\left(\frac{n \Delta}{\pi}\right)} \times \\
\times \frac{2 \mu^{2} v_{0}}{1-\frac{P_{0}}{P_{*}}} .
\end{gathered}
$$

Получены значения коэффициентов гармонического разложения (76), (77), (78). Вычисленные коэффициенты $b_{0}, a_{2}, b_{2}$ зависят от начальной неправильности труб става $v_{0}$, коэффициента возбуждения нагрузки $\mu$ отношения н оминального статического значения $P_{0}$ к критической продольной нагрузке $P$ *. Амплитуды колебаний имеют тенденцию к нарастанию по мере приближения $P_{0}$ к $P_{*}$ критической силе по Эйлеру. Амплитуда колебаний достигает максимума при $n \approx 1$ и $\Omega-p_{1} \geq 0$.

Таким образом, буровой став нагружен продольным усилием $P_{n}$, крутящим моментом $M_{\text {к }}$, под действием которого став получает угловую скорость вращения $\omega_{B}=0 \div 60$ об/мин (в ряде случаев до 150 об/мин). Став, набранный из горячекатанных труб по ГОСТ 8732-78, имеет изначально эксцентриситет в профиле 24 мм (на погонный метр). Кроме того, вследствие совместного действия усилия $P_{n}$, приложенного с эксцентриситетом, и крутящего момента $M_{\text {к, }}$ возрастает изгибающий момент, обусловленный начальной неправильностью и продольным изгибом колонны. Если же под действием возрастающих силовых факторов произойдет потеря устойчивости участка става длиной $l_{\text {кр }}$ (равной половине шага винтовой линии), то будет выбран зазор между диаметром скважины и диаметром штанги $a_{1}$, при этом возрастет сопротивление проворачиванию става, возрастет крутящий момент на вращателе, критическая длина участка става уменьшится и буровой став войдет гарантировано в неустойчивое состояние, при этом изгибные колебания става существенно возрастут. Кроме того, если фактическая частота вращения става $\omega_{\text {в }}$ совпадет с одной из собственных частот изгибных колебаний става $\omega_{k}=(k \pi / l)^{2} \sqrt{\frac{E I}{m}},(k=1,2,3,4, \ldots)$ или частотой $\Omega_{\mathrm{k}}$, то поперечный изгиб става по одной из основных форм изгибных колебаний, при отсутствии поперечных связей со стороны пробуренной выработки, будет неограниченно возрастать (см. формула (80)). В нашем случае этот изгиб $v(x)$ приведет к дополнительному сопротивлению проворачивания става, возрастанию крутящего момента $M_{\text {к }}$ и развитию продольных колебаний става под действием $\Delta P_{n}$ и повышению вибрационной нагрузки на машину в целом.

В связи с тем, что работа бурового става сопровождается изменением угловой скорости его вращения в довольно широких пределах, то имеется высокая вероятность совпадения этих угловых скоростей с собственными частотами изгибных колебаний става. Путем изменения усилия подачи $P_{\mathrm{n}}$ собственные частоты колебаний можно изменять в пределах от $\Omega_{\mathrm{k}}$ до $\omega_{\mathrm{k}}$, a также изменяя $\omega_{\text {в }}$ будет изменяться и $M_{\text {к }}$, при этом став можно вывести из неустойчивой зоны работы за счет применения специальных автоматических подстройщиков, как по $P_{n}$, так и по $\omega_{\mathrm{B}}\left(M_{\mathrm{\kappa}}\right)$, например, [1].

Снижение уровня параметрических колебаний на забой возможно за счет применения наложенных вибраций на забой с точки зрения внесения ударных импульсов с вибрационной скоростью, превышающей скорость бурения в противофазе к возникающим колебаниям на критической частоте, с целью как увеличения эффективности бурения, так и с целью ограничения совместных крутильных, продольных и поперечных колебаниях бурового става.

Дифференциальные уравнения (69) или (71) описывают параметрические и вынужденные колебания бурового става с частотой крутильной колебательной системы $p_{1}$. Коэффициенты решения $b_{0}, a_{2}, b_{2}$ также содержат частоты собственных изгибных колебаний незагруженной $\left(\omega_{\mathrm{k}}\right)$ и загруженной $\left(\Omega_{\mathrm{k}}\right)$ систем, а также коэффициент возбуждения нагрузки $(\mu)$.

Решение исходного дифференциального уравнения с правой частью допускает решение в виде (74), (76), (77), (78). Изгибные колебания происходят по закону (74) с частотой собственных крутильных колебаний двухмассовой крутильной системы, а амплитуды вынужденных колебаний определяются коэффициентами $a_{2}$, $b_{2}$.

Помимо областей неустойчивости при поперечных колебаниях, возбуждаемых параметрически за счет приращений $\Delta P_{n}\left(\Delta M_{k}\right)$, возможно возмущение поперечных колебаний от неуравновешенности става, обусловленной начальной неправильностью бурильных труб, а также изгибом става при потере им продольной устойчивости, когда частота вращения става совпадает с одной из собственных частот поперечных колебаний. Предположим, что при изги- 
бе общий прогиб става под действием продольной переменной нагрузки $P_{n}$, соотношение (59), может быть разложен по формам изгибных колебаний в виде [10]

$$
v(x)=\bar{b}_{1}, y_{1}(x)+\bar{b}_{2}, y_{2}(x)+\bar{b}_{3}, y_{3}(x)+\ldots
$$

где $\bar{b}_{1}, \bar{b}_{2}, \bar{b}_{3}-$ коэффициенты; $y_{1}(x), y_{2}(x)$, $y_{3}(x), \ldots$. - собственные формы колебаний става, как длинного стержня круглого сечения с шарнирным опиранием концов.

С учетом значений коэффициентов и вида собственных форм колебаний [12] выражение прогибов запишем в виде

$$
\begin{gathered}
v(x)=\frac{\bar{a}_{1}}{1-\frac{\omega_{B}^{2}}{\Omega_{1}^{2}}} \sin \frac{\pi}{l} x+\frac{\bar{a}_{3}}{1-\frac{\omega_{B}^{2}}{\Omega_{3}^{2}}} \sin \frac{3 \pi}{l} x+ \\
+\frac{\bar{a}_{5}}{1-\frac{\omega_{B}^{2}}{\Omega_{5}^{2}}} \sin \frac{5 \pi}{l} x+\ldots .
\end{gathered}
$$

При совпадении фактической частоты вращения става $\omega_{\mathrm{B}}$ с одной из собственных частот изгибных колебаний става (под действием усилия прижатия $\left.P_{\text {по }}\right) \Omega_{1}, \Omega_{3}, \Omega_{5}$ происходит неограниченное возрастание прогибов става от действия начального дебаланса или искривления става под действием сжимающего усилия $P_{n}$. Как показывает практика эксплуатации буровых станков изгиб става происходит, в основном, по первой главной форме колебаний. В данном случае прогибы става ограничены величиной зазора между ставом и стенками скважины $a$, которая определяется полуразностью между диаметрами скважины и штанги. При необходимости величины коэффициентов $\overline{a_{1}}, \overline{a_{2}}, \overline{a_{3}} \ldots$ подлежат определению, согласно [12].

\section{Выводы}

1. В работе установлено, что в результате действия на буровой став крутящего момента на вращателе и усилия подачи, буровой став деформируется по пространственной кривой (винтовой линии).

2. Установлено, что источником возникновения динамических состояний вращающегося бурового става является переменный момент сил трения между инструментом и забоем, который приводит к раскачке крутильных колебаний и связанных с ними продольными и попе- речными параметрическими колебаниями.

3. Определены зоны динамической неустойчивости при поперечных колебаниях става, возбуждаемых параметрически с частотой крутильных колебаний и нарастающими амплитудами при периодическом изменении приращений крутящего момента $\Delta M_{k}$ и усилия подачи $\Delta P_{n}$.

4. Выявлены зоны динамической неустойчивости при вращении деформированного става с начальными неправильностями. Эти зоны возникают при совпадении угловой скорости вращения става с одной из собственных частот изгибных колебаний.

5. Получены выражения для коэффициентов динамичности бурового става при крутильных и продольных колебаниях. Установлено, что коэффициент динамичности при продольнопоперечных процессах зависит существенно от свободного хода механизма подачи, который необходимо ограничивать в реальных машинах.

6. Рекомендовано, с целью снижения виброактивности бурового става применить следящую систему автоматического регулирования, которая подстраивает усилие подачи и скорость вращения бурового става в зонах динамической неустойчивости до получения вибрации става допустимого уровня.

7. Предложено снижать виброактивность става и повышать эффективность бурения за счет применения наложенных вибраций, оптимально подобранных по частоте и амплитуде в зоне взаимодействия инструмента с породой.

\section{Библиографический список}

1. Потураев В. Н., Равцов М. В. Основы методики инженерных расчетов машин шарошечного бурения с учетом виброколебаний. $\mathrm{Ha}$ дежность горных и транспортных машин : сб науч. трудов, Киев : Наукова думка, 1985. C. 111-116.

2. Суханов А. Ф., Кутузов Б. Н., Шмидт Р. Г. Вибрация и надежность работы станков шарошечного бурения. М. : Недра, 1969. $126 \mathrm{c}$.

3. Кучма В. В. О динамической неустойчивости бурового става станков СБШ-250. Материаль Международной научно-технической конференции «Розвиток промисловості та суспільства». Кривой Рог, 2018. Т. 2. С. 38, С. 47.

4. Вольмир А. С. Устойчивость деформируемых систем. М. : Наука, 1972. 800 с.

5. Камке Э. Справочник по обыкновенным дифференциальным уравнениям. М. : Наука, 
$1971.576 \mathrm{c}$.

6. Пановко Я. Г., Губанова И. И. Устойчивость и колебания упругих систем. М. : Наука, 1969. $420 \mathrm{c}$.

7. Малиновский Ю. А., Малиновская А. Ю., Бондарец А. А. Особенности выбора параметров и расчета упругих муфт в приводах крупных мельниц для измельчения минерального сырья. Сб. науч. тр. по материалам Международной научно-практической конферениии. Современные направления теоретических и прикладных исследований. Одесса, 2009. Т.7. Технические науки. С. 34-39.

8. Хвингия М. В. Вибрации пружин. М. : Машиностроение, 1966. 287 с.

9. Вибрации в технике : справочник в 6-ти томах / Т.1 под ред. Болотина В. В. М. : Машиностроение, 1978. $352 \mathrm{c}$.

10. Филоненко-Бородич М. М., Изюмов С. М., Олисов Б. А. и др. Курс сопротивления материалов. Ч. 2. М. : Физматгиз, 1962. 528 с.

11. Попов С. О., Малиновский Ю. А., Малиновская С. И. и др. Некоторые причины возникновения вибраций бурового става при работе карьерных станков шарошечного бурения. Металлургическая и горнорудная промышленность. 2016. № 2. С. 95-100.

12. Биргер И. А., Шорр Б. Ф., Иосилевич Г. Б. Расчет на прочность деталей машин : справочник. М. : Машиностроение, 1979. 702 с.

\section{References}

1. Poturaev V. N., Ravtsov M. V. (1985). Osnovy metodiki inzhenernykh raschetov mashin sharoshechnogo bureniia s uchetom vibrokolebanii. Nadezhnost gornykh i transportnykh mashins: $s b$ nauch. trudov, Kiev: Naukova dumka, 111-116

2. Sukhanov A. F., Kutuzov B. N., Shmidt R. G. (1969). Vibratsiia i nadezhnost raboty stankov sharoshechnogo bureniia. Moskva: Nedra

3. Kuchma V. V. (2018). O dinamicheskoi neustoichivosti burovogo stava stankov SBSH-250. Materialy Mezhdunarodnoi nauchno-tekhnicheskoi konferentsii "Rozvytok promyslovosti ta suspilstva". Krivoi Rog, 2

4. Volmir A. S. (1972). Ustoichivost deformiruemykh sistem. M. : Nauka

5. Kamke Eh. (1971). Spravochnik po obyknovennym differentsialnym uravneniiam. Moskva: Nauka

6. Panovko Ia. G., Gubanova I. I. (1969). Ustoichivost i kolebaniia uprugikh sistem. Moskva: Nauka

7. Malinovskii Iu. A., Malinovskaia A. Iu., Bondarets A. A. (2009). Osobennosti vybora parametrov i rascheta uprugikh muft $\mathrm{v}$ privodakh krupnykh melnits dlia izmelcheniia mineralnogo syria. Sb. nauch. tr. po materialam Mezhdunarodnoi nauchno-prakticheskoi konferentsii. Sovremennye napravleniia teoreticheskikh $i$ prikladnykh issledovanii. Odessa, 7. Tekhnicheskie nauki, 34-39

8. Khvingiia M. V. (1966). Vibratsii pruzhin. Moskva: Mashinostroenie

9. Bolotina V. V. (Ed.) (1978). Vibratsii v tekhnike. Moskva: Mashinostroenie

10. Filonenko-Borodich M. M., Iziumov S. M., Olisov B. A. (1962). Kurs soprotivleniia materialov, 2. Moskva: Fizmatgiz

11. Popov S. O., Malinovskii Iu. A., Malinovskaia S. I. (2016). Nekotorye prichiny vozniknoveniia vibratsii burovogo stava pri rabote karernykh stankov sharoshechnogo bureniia. Metallurgicheskaia $i$ gornorudnaia promyshlennost, (2), 95-100.

12. Birger I. A., Shorr B. F., Iosilevich G. B. (1979). Raschet na prochnost detalei mashin : spravochnik. Moskva: Mashinostroenie

Учитель Олександр Давидович, професор, доктор технічних наук, Національна металургійна академія України (м. Дніпро, Україна). E-mail: ODUchitel@i.ua

Маліновський Юрій Олександрович, доцент, кандидат технічних наук, Криворізький коледж Національного авіаційного університету (м. Кривий Ріг, Україна). E-mail: malinovsky129@gmail.com

Власенков Дмитро Петрович, старший викладач, Криворізький коледж Національного авіаційного університету (м. Кривий Ріг, Україна). E-mail: Vlasenkov.d@gmail.com

Кравчук Ігор Миколайович, викладач, Криворізький коледж Національного авіаційного університету (м. Кривий Ріг, Україна). E-mail: igor-kravchuk77@ukr.net

Терьошина Світлана Сергіївна, викладач, Криворізький коледж Національного авіаційного університету (м. Кривий Ріг, Україна). E-mail: Svetlana_tereshi@ukr.net 


\section{ПРИЧИНИ ВИНИКНЕННЯ ДИНАМІЧНОЇ НЕСТІЙКОСТІ БУРОВОГО СТАВУ ПРИ РОБОТЕ ВЕРСТАТІВ СВШ- 250}

Мета - розробка науково-обгрунтованої методики розрахунку бурових верстатів типу СВШ-250, що дозволяє визначити критичні навантаження бурового става, що викликають динамічно нестійкі стани системи: буровий інструмент - став - обертач - щогла - машинне відділення. Крім того, намітити шляхи щодо зменшення динамічних складових крутного моменту на обертачі і зусилля притиснення бурового става.

Методика. Для обгрунтування причин динамічно нестійкої роботи верстата прийнято уявлення про те, що буровий став, який обертається під дією крутного моменту і зусилля подачі при бурінні свердловини набуває форму гвинтової лінії, який знаходиться в динамічно нестійкому стані, викликаним моментом сил тертя між буровим інструментом і забоєм. Складено відповідні системи диференційних рівнянь і рівняння балансу кінетичної і потенційної енергій системи верстата при коливаннях.

Результати. У роботі встановлено, що в результаті дії на буровій став крутного моменту і зусилля подачі став деформується і при певних значеннях силових факторів став втрачає подовжню стійкість і набуває форму гвинтової лінії, «накрученою» на свердловину. У цьому випадку робота става і всього верстата супроводжується значними вібраціями. Проведені дослідження дозволили визначити, що джерелом як крутильних, поздовжніх, так і згинальних коливань става є нелінійне тертя між інструментом і забоєм. Крім того, авторами виявлені зони динамічної нестійкості при роботі става і намічений ряд практичних рекомендацій для стабілізації роботи става і всього верстата в цілому.

Наукова новизна роботи полягає в тому, що виявлено джерело складних поздовжньозгинально-крутильних коливань бурового става, яким $є$ нелінійна характеристика тертя між забоєм і інструментом, що володіє спадаючою ділянкою. У зв'язку з цим фрикційні крутильні автоколивання викликають поздовжньо-ізгібні параметричні коливання, які порушуються з частотою власних коливань крутильної системи. Крім того, встановлено, що збіг власних частот згинальних коливань 3 частотою обертання $є$ причиною нестійкості при роботі става.

Практична цінність роботи полягає в тому, що виявлені зони динамічної нестійкості поздовжньо-згинальних коливань бурового става, які відповідають власним частотам і формам згинальних коливань під дією збільшення поздовжнього зусилля подачі. У разі збігу однієї з власних частот згинальних коливань 3 кутовою частотою обертання става настає необмежене зростання амплітуд згинальних коливань, зростання яких стримується зазором між діаметрами свердловини та штанги. Розглядаючи окремо енергетику крутильних і поздовжніх коливань, отримані вирази для коефіцієнтів динамічності. Встановлено, що поздовжній коефіцієнт динамічності при однаковому статичному вкороченні става приблизно пропорційний кореню квадратному з величини вільного ходу бурового става (тобто вільний хід става необхідно обмежити). Рекомендовано з метою зниження вібраційної активності бурового става застосовувати систему автоматичного регулювання кутової частоти обертання, яка самостійно налаштовується, спільно із зусиллям подачі става. Крім того, рекомендується зниження вібраційної активності за рахунок застосування накладених вібрацій в зоні взаємодії інструменту 3 забоєм.

Ключові слова: буровий став, долото, обертач, механізм подачі штанг, вібростійкість, критична сила, критична довжина става.

Oleksandr Uchytel, Professor, Doctor of Technical Sciences, National Metallurgical Academy of Ukraine (Dnipro, Ukraine). E-mail: ODUchitel@i.ua

Yurii Malinovskyi, Associate Professor, Candidate of Technical Sciences, Kryvyi Rih College of National Aviation University (Kryvyi Rih, Ukraine). E-mail: malinovsky129@gmail.com

Dmytro Vlasenkov, Senior Lecturer, Kryvyi Rih College of the National Aviation University (Kryvyi Rih, Ukraine). E-mail: Vlasenkov.d@gmail.com

Ihor Kravchuk, Lecturer, Kryvyi Rih College of the National Aviation University (Kryvyi Rih, Ukraine). E-mail: igor-kravchuk77@ukr.net

Svitlana Teroshyna, Lecturer, Kryvyi Rih College of the National Aviation University (Kryvyi Rih, 
Ukraine).E-mail: Svetlana_tereshi@ukr.net

\section{CAUSES OF DYNAMIC INSTABILITY OF THE DRILLING ROD DURING THE OPERATION OF MACHINES SBSh-250}

The purpose of the work is the development of a scientifically grounded methodology for calculating drilling rigs of the SBSh-250 type, which makes it possible to determine the critical loads of the drilling string that cause dynamically unstable states of the system: drilling tool - stand - rotator - mast - engine room. In addition, outline the ways to reduce the dynamic components of the rotator torque and the pressing force of the drilling rod.

Methods. To substantiate the reasons for the dynamically unstable operation of the machine, it is assumed that a rotating drill rod under the action of a torque and feed force while drilling a well takes the form of a helix, which is in a dynamically unstable state caused by the frictional moment between the drilling tool and the bottomhole. The corresponding systems of differential equations and balance equations for the kinetic and potential energies of the machine tool system during oscillations have been compiled.

Results. It has been established in the work that as a result of the action of the torque and feed force on the drill rod, the steel becomes deformed and, at certain values of the force factors, the steel loses its longitudinal stability and acquires the shape of a helical line "wound" onto the well. In this case, the work of the drilling rod and the entire machine is accompanied by significant vibrations. The studies carried out made it possible to determine that the source of both torsional, longitudinal, and bending vibrations of the rod is nonlinear friction between the tool and the bottom. In addition, the authors have identified zones of dynamic instability during the work of the mill and outlined a number of practical recommendations for stabilizing the work of the mill and the entire machine as a whole.

Originality of work lies in the fact that the source of complex buckling-torsional vibrations of the drilling rod has been identified, which is a nonlinear characteristic of friction between the bottomhole and the tool, which has a falling section. In this regard, frictional torsional self-oscillations cause longitudinalbending parametric vibrations, which are excited at the natural frequency of the torsion system. In addition, it was found that the coincidence of the natural frequencies of bending vibrations with the rotation frequency is the cause of instability during the operation of the rod.

Practical implications of work consists in the fact that the zones of dynamic instability of the longitudinal-flexural vibrations of the drilling rod are identified, which correspond to the natural frequencies and forms of bending vibrations under the action of the increment of the longitudinal feed force. If one of the natural frequencies of bending vibrations coincides with the angular frequency of rotation of the drilling rod, an unlimited increase in the amplitudes of bending vibrations occurs, the growth of which is restrained by the gap between the diameters of the well and the rod. Considering separately the energetics of torsional and longitudinal vibrations, expressions for the dynamic factors are obtained. It has been established that the longitudinal dynamic coefficient with the same static shortening of the drilling rod is approximately proportional to the square root of the free stroke of the drilling string (i.e., the free stroke of the drilling rod must be limited). Recommended to reduce the vibration activity of the drill string use a self-adjusting system for automatic control of the angular rotation frequency together with the feed force of the drilling rod. In addition, it is recommended to reduce vibration activity by applying superimposed vibrations in the zone of interaction of the tool with the bottom.

Key words: drilling rod, bit, rotator, rod feed mechanism, vibration resistance, critical force, critical length of the drilling rod.

Учитель Александр Давыдович, профессор, доктор технических наук, Национальная металлургическая академия Украины (г. Днепр, Украина). E-mail: ODUchitel@i.ua

Малиновский Юрий Александрович, доцент, кандидат технических наук, Криворожский колледж Национального авиационного университета (г. Кривой Рог, Украина). E-mail: malinovsky129@gmail.com

Власенков Дмитрий Петрович, старший преподаватель, Криворожский колледж Национального авиационного университета (г. Кривой Рог, Украина). E-mail: Vlasenkov.d@gmail.com

Кравчук Игорь Николаевич, преподаватель, Криворожский колледж Национального авиаци- 
онного университета (г. Кривой Рог, Украина). E-mail: igor-kravchuk77@ukr.net

Терешина Светлана Сергеевна, преподаватель, Криворожский колледж Национального авиационного университета (г. Кривой Рог, Украина). E-mail: Svetlana tereshi@ukr.net

\section{ПРИЧИНЫ ВОЗНИКНОВЕНИЯ ДИНАМИЧЕСКОЙ НЕУСТОЙЧИВОСТИ БУРОВОГО СТАВА ПРИ РАБОТЕ СТАНКОВ СБШ-250}

Цель - разработка научно-обоснованной методики расчета буровых станков типа СБШ-250, позволяющей определить критические нагрузки бурового става, вызывающие динамически неустойчивые состояния системы: буровой инструмент - став - вращатель - мачта - машинное отделение. Кроме того, наметить пути по уменьшению динамических составляющих крутящего момента на вращателе и усилия прижатия бурового става.

Методика исследований. Для обоснования причин динамически неустойчивой работы станка принято представление о том, что вращающийся буровой став под действием крутящего момента и усилия подачи при бурении скважины приобретает форму винтовой линии, который находится в динамически неустойчивом состоянии, вызванным моментом сил трения между буровым инструментом и забоем. Составлены соответствующие системы дифференциальных уравнений и уравнения баланса кинетической и потенциальной энергий системы станка при колебаниях.

Результаты. В работе установлено, что в результате действия на буровой став крутящего момента и усилия подачи став деформируется и при определенных значениях силовых факторов став теряет продольную устойчивость и приобретает форму винтовой линии, «навитой» на скважину. В этом случае работа става и всего станка сопровождается значительными вибрациями. Проведенные исследования позволили определить, что источником как крутильных, продольных, так и изгибных колебаний става является нелинейное трение между инструментом и забоем. Кроме того, авторами выявлены зоны динамической неустойчивости при работе става и намечен ряд практических рекомендаций для стабилизации работы става и всего станка в целом.

Научная новизна работы заключается в том, что выявлен источник сложных продольноизгибно-крутильных колебаний бурового става, которым является нелинейная характеристика трения между забоем и инструментом, обладающая ниспадающим участком. В этой связи фрикционные крутильные автоколебания вызывают продольно-изгибные параметрические колебания, которые возбуждаются с частотой собственных колебаний крутильной системы. Кроме того, установлено, что совпадение собственных частот изгибных колебаний с частотой вращения является причиной неустойчивости при работе става.

Практическая ценность работы заключается в том, что выявлены зоны динамической неустойчивости продольно-изгибных колебаний бурового става, которые соответствуют собственным частотам и формам изгибных колебаний под действием приращения продольного усилия подачи. В случае совпадения одной из собственных частот изгибных колебаний с угловой частотой вращения става наступает неограниченное возрастание амплитуд изгибных колебаний, рост которых сдерживается зазором между диаметрами скважины и штанги. Рассматривая отдельно энергетику крутильных и продольных колебаний, получены выражения для коэффициентов динамичности. Установлено, что продольный коэффициент динамичности при одинаковом статическом укорочении става примерно пропорционален корню квадратному из величины свободного хода бурового става (т.е. свободный ход става необходимо ограничить).

Рекомендовано с целью снижения виброактивности бурового става применять самонастраивающуюся систему автоматического регулирования угловой частоты вращения совместно с усилием подачи става. Кроме того, рекомендуется снижение виброактивности за счет применения наложенных вибраций в зоне взаимодействия инструмента с забоем.

Ключевые слова: буровой став, долото, вращатель, механизм подачи штанг, виброустойчивость, критическая сила, критическая длина става. 